\title{
Information and Communications Systems for Control-by-Price of Distributed Energy
} Resources and Flexible Demand

Nyeng, Preben; Østergaard, Jacob

Published in:

IEEE Transactions on Smart Grid

Link to article, DOI:

10.1109/TSG.2011.2116811

Publication date:

2011

Link back to DTU Orbit

Citation $(A P A)$ :

Nyeng, P., \& Østergaard, J. (2011). Information and Communications Systems for Control-by-Price of

Distributed Energy Resources and Flexible Demand. IEEE Transactions on Smart Grid, (1949-3053).

https://doi.org/10.1109/TSG.2011.2116811

\section{General rights}

Copyright and moral rights for the publications made accessible in the public portal are retained by the authors and/or other copyright owners and it is a condition of accessing publications that users recognise and abide by the legal requirements associated with these rights.

- Users may download and print one copy of any publication from the public portal for the purpose of private study or research.

- You may not further distribute the material or use it for any profit-making activity or commercial gain

- You may freely distribute the URL identifying the publication in the public portal

If you believe that this document breaches copyright please contact us providing details, and we will remove access to the work immediately and investigate your claim 


\title{
Information and Communications Systems for Control-by-Price of Distributed Energy Resources and Flexible Demand
}

\author{
Preben Nyeng, Member, IEEE, and Jacob Østergaard, Senior Member, IEEE
}

\begin{abstract}
The control-by-price concept fits well with controlling small-scale generation, storage and demand. In this paper, we investigate the required information and communications systems that are needed to realize the control-by-price concept for such units. We first present a proposal for overall infrastructure and subsystem design and secondly focus on the design and implementation of the end-user price-responsive controller, interfaces, and communications. The design and its applicability on existing devices is verified through laboratory tests with two cases: electric space heating thermostat control and a small combined heat and power unit. The results show that the price-responsive controller reduces the end user's electricity cost, or increases his income respectively, by about $7 \%$. At the same time, the price-responsive controller provides an interface for the transmission system operator to utilize distributed energy resources and flexible demand as a regulating resource. Furthermore, the results illustrate and verify the applicability of the concept and the proposed infrastructure for controlling distributed energy resources and flexible demand.
\end{abstract}

Index Terms-Control-by-price, distributed energy resources, micro-CHP, power system balancing, real-time market.

\section{INTRODUCTION}

$\mathbf{S}$ INCE Schweppe et al.'s seminal paper on Homeostatic Utility Control [1], the fundamental idea of letting smallscale generation, load, and storage respond to the instantaneous electricity price has been investigated and is still a subject for research and demonstration projects. With the increasing penetration of intermittent generation in the power system, the need for utilizing all available resources for power system balancing arises and thus the need for a system that can control potentially millions of individual units on a time-scale in the order of a few minutes.

On the Olympic Peninsula, Washington, USA, a demonstration project has been conducted with a local 5-min market comprising loads as well as small generators. The results are very promising and show a significant influence of the real-time price on consumers as well as small-scale producers of power [2].

This market concept uses an auction and bidding process prior to each 5-min market interval to find the price. In our work we focus on a concept that is closer to the initial proposal in [1],

Manuscript received September 28, 2010; revised February 01, 2011; accepted February 12, 2011. Paper no. TSG-00141-2010.

The authors are with the Centre for Electric Technology, Technical University of Denmark (e-mail: pny@elektro.dtu.dk; joe@elektro.dtu.dk).

Color versions of one or more of the figures in this paper are available online at http://ieeexplore.ieee.org.

Digital Object Identifier 10.1109/TSG.2011.2116811 in which an electricity price is determined by the system operator every 5 minutes based on the actual condition of the power system and transmitted to the customers (which can either be net consumers or net producers). General feasibility analyses of the concept can be found in [3] and [4].

This "control-by-price" concept is well suited to control many small units, due to its extreme transparency and simplicity. It implies that the price must be sent to the end user in due time and that a computer can receive the price and take appropriate control actions that maximize the end user's profit. The concept is particularly useful for units that are too small to participate in the conventional power system control schemes, e.g., distributed energy resources (DER), which is small-scale generation or storage and electric loads that inherently have the ability to be postponed or otherwise rescheduled, such as heating and cooling applications, or electric vehicle chargers.

In this paper, we investigate the required information and communications systems that are needed to realize the concept. In the following subsections, we propose an overall system architecture and the associated communications systems and protocols. In Section II, we describe the design and implementation of a price-responsive controller and the laboratory setup we have used to verify the functionality of the controller and infrastructure. Finally we show laboratory results, obtained using the controller in connection with two different applications.

\section{A. Proposed System Architecture and Infrastructure}

In Fig. 1 an overview of the proposed system architecture and infrastructure is shown. The price is calculated, based on input from the system operator about the present system conditions and especially the need for balancing control actions. The price is then sent real-time to the end users that have price-responsive controllers connected to DER units or controllable loads. For domestic applications this would typically be small combined heat and power units (micro-CHP), heat pumps, electric vehicle chargers, or any other device that is able to reschedule, reduce, or increase generation or demand according to the electricity price. This in turn affects the power balance in the electricity network, which is taken into account when the next price is to be calculated. This way the left part of Fig. 1 constitutes a closedloop control system for power system balancing. In addition to this an offline settlement is needed, depicted in the right half of Fig. 1. Even though this process does not directly affect the closed-loop performance, it is necessary to ensure that the priceresponsive controller and its owner has the right incentives to respond to the price as a control signal. 


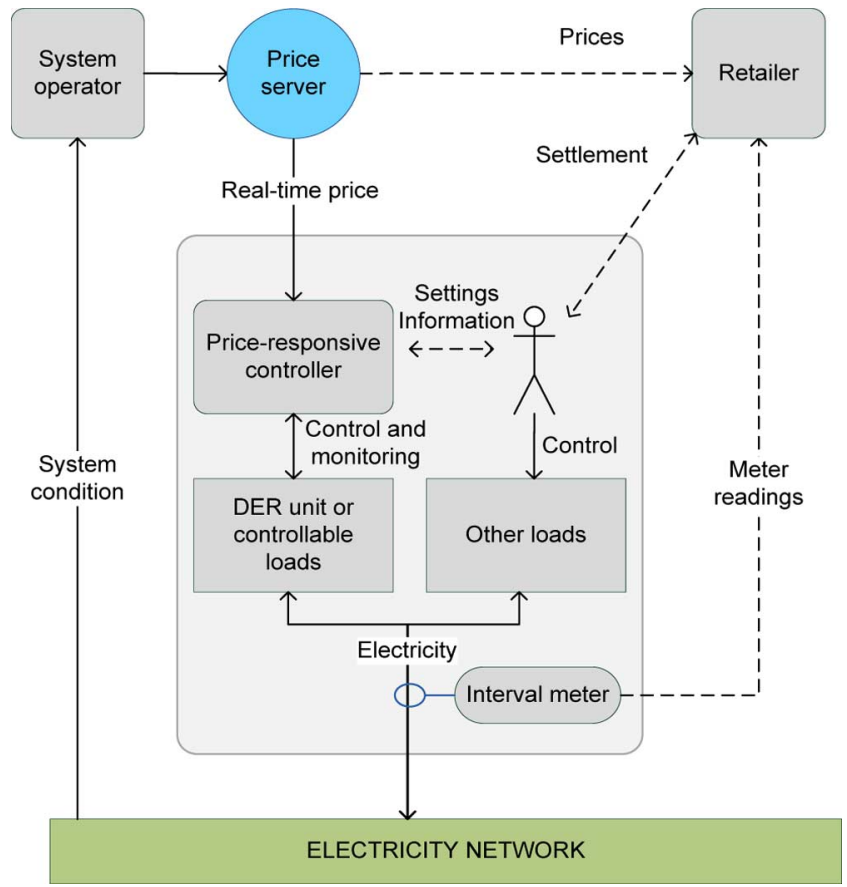

Fig. 1. System architecture and infrastructure overview. The dashed lines illustrate offline processes.

The price-responsive controllers are central for the concept. These controllers receive the current price and take according control actions and thereby create the link from system operator to balancing resource. In addition, the controllers must usually be able to receive settings and preferences from the end user and provide real-time and historical information about price and device operation to the user.

In Section II we will consider the detailed design of a priceresponsive controller and in particular the algorithms used to decide what actions to take in connection with specific devices.

1) Metering: The response to a price update in terms of adapting the generation or demand, can be metered together with the other loads (and/or generation) in the building. In contrast to conventional balancing services, like the so-called regulating power in the Nordic market context, there is no need to monitor individual units and their response to assure that the response corresponds to what has been promised. In the regulating power market, the participants make bids prior to any control actions being taken and there is a financial penalty if one does not comply with ones bid. This means that the TSO needs to monitor every single unit, to see if it really responds when the TSO requests it to do so. However, in the control-by-price concept there are no bids and therefore no need to monitor individual units to see if they comply with the bids. What really matters is the total system balance that the TSO monitors through the grid frequency and intertie exchange. The obvious drawback of this compared with the regulating power market is that the TSO has no guarantee of a certain response to a given price change. However, due to the expected large number of participants in the system, statistics will provide this knowledge to the TSO.

For the control-by-price to work, it must be assured that the customers are subject to the prevailing real-time price. To assure this, the customer's electricity meter must be able to mea-

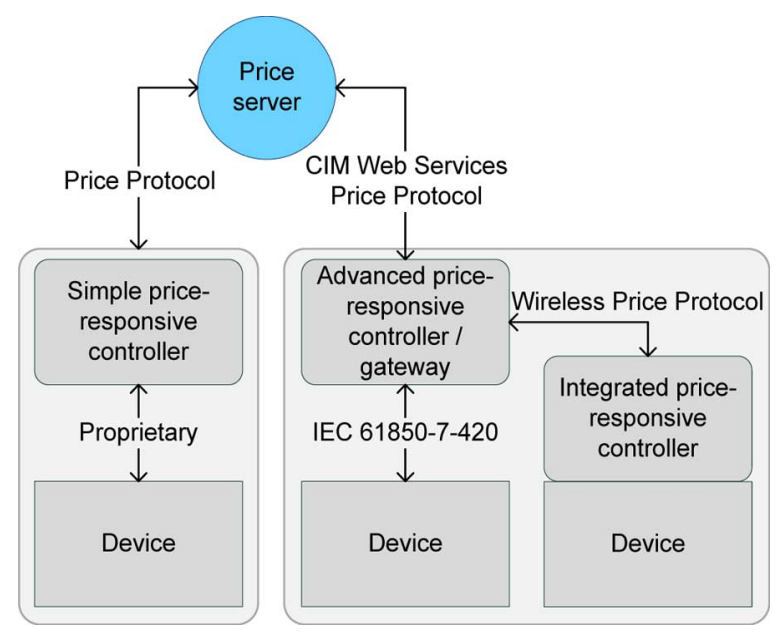

Fig. 2. Protocols and standards overview and examples of their application. The structure allows for price-responsive controllers to act as gateways and forward price information to other controllers, by, e.g., wireless media like ZigBee or Z-Wave.

sure and store meter reading with the same time resolution as the real-time price. This can be 5 or maybe 15 min intervals, with previous examples tending to prefer the $5 \mathrm{~min}$ interval [1], [2]. This implies a significant increase in the amount of information that must be transferred from the meter to the retailer, compared with the present situation where domestic meters usually supply one reading per year and the choice of time resolution is a trade-off between data rates and response time when considering the TSO's need for balancing resources.

An alternative solution is to integrate the product of price and electricity quantity locally in the meter and transfer the resulting net value for settlement. However, this also limits the possibilities for adding taxes and grid tariffs in a flexible manner and introduces problems, such as what the meter should do if the price is not available.

\section{B. Communications}

The most critical communications link is the one between the price server and the end-user site. The information is basically a unidirectional message and broadcast media, e.g., radio signals could be used. However, with Internet connections being readily available in many homes, utilizing this existing infrastructure seems more feasible. For easy adaptation, the protocols in use should be standardized, so that manufacturers of price-responsive products would not have to implement separate protocols for each system they want their products to be integrated in. An overview of the proposed structure is shown in Fig. 2 and described in detail in the following sections.

1) The Price Protocol: In its most simple form, the concept only requires the prevailing price to be broadcast to all units. Like in the mail system there is no broadcast function in the Internet addressing system, so the price clients would all have to retrieve the price individually. The protocol used should imply the least possible overhead and processing requirements, which points towards a UDP-based protocol, inspired by, e.g., time server requests and name server lookups. In our case, we have implemented a very simple protocol, in which the client sends an empty UDP datagram to a server, which in turn replies with 
a single datagram containing the current price. This "Price Protocol" copies the design of the Time Protocol [5] and requires the least possible overhead and processing and can thus be implemented on virtually any platform, even low-cost microcontroller platforms with very limited performance. This protocol requires totally 60 bytes of data to be transferred between the client and the server for each request. To illustrate the bandwidth requirement at the server side, assume there are 1 million clients that need to retrieve the new price within a $10 \mathrm{~s}$ time frame. The average data transfer rate at the server side is thus $48 \mathrm{Mbit} / \mathrm{s}$ plus possible media overhead, which is clearly within the limits of a fiber connection. If more clients participate in the system, the bandwidth requirements can be reduced by using mirrored servers.

2) Complex Price Information: The above protocol fulfils the absolute minimum requirement, but to improve the operation of individual units, more information is useful. The day-ahead price can for example be sent to the clients as well and serve as a forecast of the real-time price to come. Furthermore, historical real-time prices could be published by the price server, to assist in the decision making process in the client. Finally, it would be desirable to retrieve more static configuration information, such as the timely resolution of the price, the timeline for publishing of the price, the currency, etc. This would allow a generic client to configure itself for several markets with different configurations.

Evidently, such complex information calls for a standardized data structure and, e.g., the common information model (CIM), specified in [6] and [7], could provide a standardized foundation for this. It fits well in the internet architecture and is from the beginning based on XML formatting, that eases interoperability. All of the above information could be formatted with CIM and allow the client to seamlessly retrieve useful configuration and operational information. However, if this is done every $5 \mathrm{~min}$ it would significantly increase the transferred amounts of data and thus increase the bandwidth requirements, so the preferred solution might be to use CIM for infrequent updates, e.g., once per day and then use the Price Protocol to get the current price.

3) DER Interface: Finally, the interface between the price-responsive controller and DER devices needs attention. This is most often using proprietary protocols, defined by the manufacturer of the DER device, but standardization would allow for a smoother adaptation of price-responsive controllers. The international standard IEC 61850-7-420 is an example of recent standardization work on DER communications [8]. This standard includes information models and communication protocol specifications for a range of DER devices. Its fundamental SCADA-like character aims at exposing status and control values to a remote party, which is exactly what the price-responsive controller needs to optimize operation according to real-time prices. However, its complex structure adds significant overhead to the communication and the processing requirements connected with this does not fit well with the need for implementing low-cost microcontrollers in the DER units as well as in the price-responsive device. However, recent adaptation of the IEC 61850 principle to a lower communications complexity level, could indicate a future path in

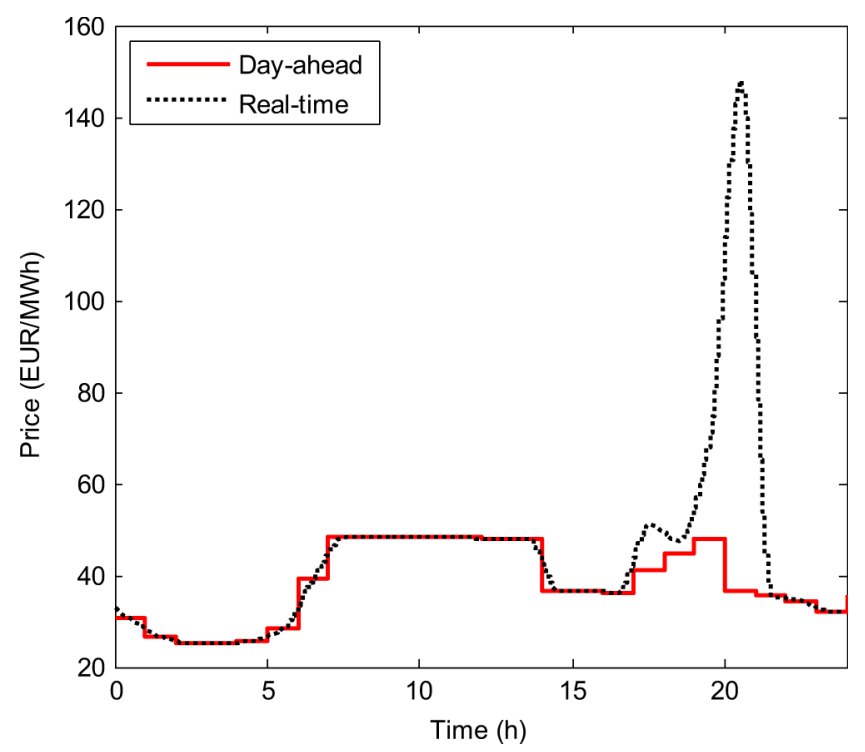

Fig. 3. Price signal and the day-ahead price. Data are from 25 September 2009, for the price zone DK1. The real-time price is created by interpolating regulating power prices.

this direction [9] and facilitate the widespread adaptation of generic price-responsive controllers, that can control a range of different processes, as long as they are IEC 61850 compliant. However, as we describe in the following sections, the control algorithms needed to control different applications may be different and require detailed information about the process characteristics and constraints.

\section{METHOD}

Based on the proposed system architecture and infrastructure, we have designed and implemented a price-responsive controller for two different applications. In this section we first introduce the general price response control algorithm and secondly, give a description of the two applications, their interfaces, laboratory setup, and specific application-dependent design of control algorithms that decide necessary control actions according to the real-time price.

\section{A. Price Signal}

At present, the real-time price is not published so we have created a time series of real-time prices, based on hourly regulating power prices published by the Danish transmission system operator [10]. We have used $24 \mathrm{~h}$ of data from 25 September 2009 , for the price zone DK1. We use the regulating power price because it indicates the balancing cost for the hour, which is the marginal cost of the balancing service procured by the TSO and thereby expresses the real-time price. Since it is only published by the hour, we interpolate the values to find 5-min interval prices. In Fig. 3 the real-time price signal is shown together with the day-ahead price for the same day. We have chosen this particular day because the overall pattern follows that of a typical day and at the same time exhibits a price spike, that occurs quite often in the Nordic regulating power market and should have significant impact on the price-responsive control. 


\section{B. Control Algorithm}

The control algorithm used to implement the price response decision is fundamentally the same, whether it is used for the micro-CHP or the space heating thermostat. In both cases, it is mostly a matter of when they operate, rather than how much. Looking at the micro-CHP, for example, it must cover the heat demand of the building (assuming there is no alternative source of heat) and has no feedback from the building to assure that the temperature is right. It therefore has to maintain a predefined minimum temperature in the heating circuit, which in turn means that it has to operate with a certain average duty cycle. However, the timing of the operation cycles can to a certain extend be rescheduled to periods with a high electricity price to maximize profit. The absence of feedback from the building with regards to temperature means that the heat storage available for price flexibility is limited to the water tank that is an integrated part of the heating circuit. Using the building as heat storage as well, would increase the potential but also complicate the system significantly. If a larger heat capacity was needed, it would probably be more feasible to simply use a larger water tank.

In contrast, the electric space heating system does not have a water tank at its disposal, so it must utilize the heat capacity of the building. The thermostats can reduce demand by lowering the temperature, but the acceptable temperature deviation compared with the inside and outside temperature difference is often negligible. The largest profit potential is also here to reschedule demand, rather than try to reduce it, by utilizing the large heat capacity in the home.

1) Relative Price: With the wish for reacting on high or low prices, the problem with defining high and low emerges. If high and low relate to an absolute value, i.e., monetary unit per energy quantity, it would impact the operation of the devices if the price for a long period was above or below the absolute thresholds.

Therefore, inspired by the use of the $24 \mathrm{~h}$ sliding window mean price and standard deviation in [2], we introduce the dimensionless, relative price $P_{\text {rel }}$ as the input price to the decision making process, defined by

$$
P_{\text {rel }} \equiv \frac{P-P_{\mathrm{avg}}}{P_{\mathrm{dev}}}
$$

$P$ is the present price and $P_{\text {avg }}$ and $P_{\text {dev }}$ are determined recursively for each time step $\Delta t$ by

$$
\begin{aligned}
P_{\mathrm{avg}, i}= & P_{\mathrm{avg}, i-1}+\frac{\Delta t}{\Delta t+\tau} \cdot\left(P-P_{\mathrm{avg}, i-1}\right), \\
P_{\mathrm{var}, i}= & P_{\mathrm{var}, i-1}+\frac{\Delta t}{\Delta t+\tau} \\
& \cdot\left(\left(P-P_{\mathrm{avg}, i}\right)^{2}-P_{\mathrm{var}, i-1}\right),
\end{aligned}
$$

and

$$
P_{\mathrm{dev}, i}=\sqrt{P_{\mathrm{var}, i}}
$$

$\tau$ is a time constant that influences how long a price change affects the relative price and thereby how fast the relative price adapts to price changes. Fig. 4 illustrates how different values of $\tau$ impact the relative price with regards to magnitude and

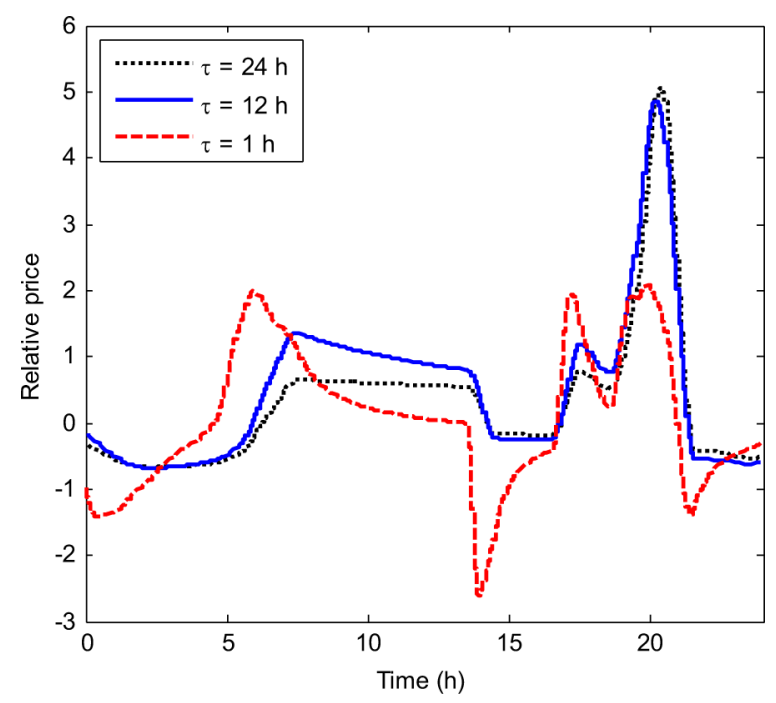

Fig. 4. Relative prices with different values of the time constant $\tau$. The relative price is calculated as a function of the absolute price from Fig. 3.

duration of price variations. A short time constant gives a lively curve with a rapidly increasing and decreasing price, that on the other hand faster descents towards a neutral price.

Compared with the sliding average method in [2], the relative price definition includes less statistical weight from the oldest samples, which means that there is no impact of historical values sliding out of the window. In addition it has the advantage with regards to implementation, that it can be calculated continuously without maintaining 24 sliding hours of data. However, upon controller reset the calculation still needs initialization values of $P_{\text {avg }}$ and $P_{\text {var }}$, or the previously calculated values must be stored in nonvolatile memory. In each of our laboratory tests, we initialize $P_{\text {avg }}$ and $P_{\text {var }}$ as if the controller had been in operation for several days, each day with the same price time series as in the actual test.

\section{Micro Combined Heat and Power (Micro-CHP)}

The first design case is for the DACHS micro-CHP unit from Senertec. This unit comprises an engine that drives a generator and can thereby generate electricity. The engine is cooled with water, that is circulated in the building for space heating. Compared with a traditional furnace this process extracts more exergy from the primary energy, which is a more useful form of energy and it is in that sense more efficient. The unit is designed to fit in a normal to large residential home, in combination with a heat storage tank, or in smaller industrial buildings as supplemental heat. The key figures for the unit are listed in Table I.

The device has an RS232 connection that is used for external monitoring and in addition, it can be started or stopped by the use of an additional relay. The machine can generally either be started or stopped and there is no option for throttling the engine. An overview of the micro-CHP subsystem is shown in Fig. 5.

1) Heat Storage Emulation: We do not actually have the heat storage tank in the laboratory, but use forced convection caloriferes to dissipate the heat to the surroundings. To perform the test as realistically as possible with the available equipment, we have emulated the heat storage tank and the building heating circuit in the controller. To express the thermal energy stored 
TABLE I

MICRO-CHP CHARACTERISTICS

\begin{tabular}{|l|l|}
\hline Fuel & $\begin{array}{l}\text { Natural gas, liquefied petroleum gas, } \\
\text { fuel oil, or biodiesel (depends on variant) }\end{array}$ \\
\hline Electric power & $5.0 . .5 .5 \mathrm{~kW}$ (depends on variant) \\
\hline Heating power & $10.5 . .12 .5 \mathrm{~kW}$ (depends on variant) \\
\hline Generator & 2-pole induction generator, water-cooled \\
\hline Engine & 1-cylinder piston engine, direct generator drive \\
\hline Start-up time, typical & 90 seconds \\
\hline Shut-down time & $<1$ second \\
\hline Minimum on-time & 30 minutes \\
\hline Interface, monitoring & RS 232, request-response protocol \\
\hline Interface, control & Relay switch (no throttle) \\
\hline
\end{tabular}

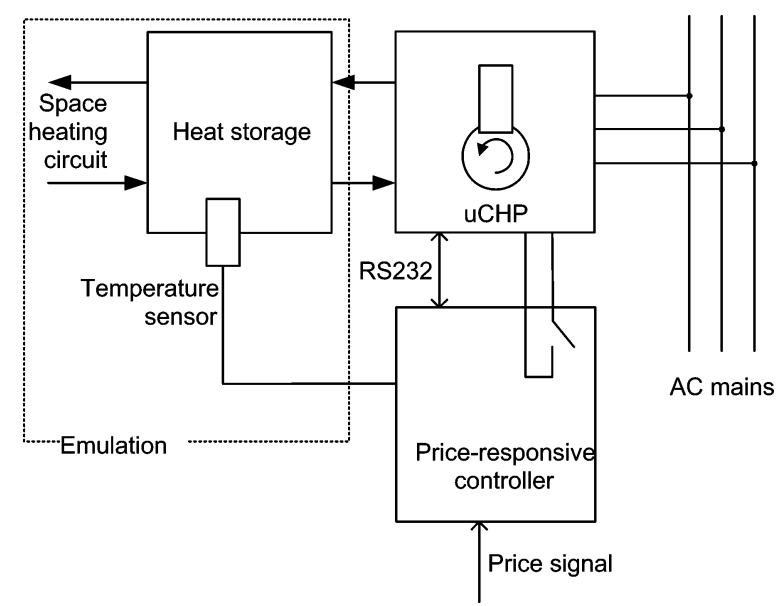

Fig. 5. Overview of micro-CHP subsystem. The price-responsive controller obtains status information through the RS232 connection and control start and stop actions with the relay. In addition it monitors the temperature in the heat storage buffer, for system optimization. The heat storage is emulated according to (5)

in the tank, we use a single temperature and assume even temperature distribution in the tank. A real storage tank could have several temperature sensors and have temperature layers, however, the average temperature would still be a valid expression for the energy state.

The temperature in the heat storage $T_{\mathrm{hs}}$ is assumed to follow

$$
\frac{d T_{\mathrm{hs}}}{d t}=\frac{\dot{Q}_{\mathrm{CHP}}-\dot{Q}_{\mathrm{demand}}}{C_{\mathrm{hs}}}
$$

where $\dot{Q}_{\mathrm{CHP}}$ is the heat flow generated by the micro-CHP unit, $\dot{Q}_{\text {demand }}$ is the heat flow to the building and $C_{\mathrm{hs}}$ is the heat capacity, or thermal mass, of the storage tank. This assumption does not take the heat loss from the heat storage to the surroundings into account, because it is assumed to be negligible compared with the heat demand. We assume that the unit delivers its rated heat flow when it signals through the RS232 interface that it is running. In reality this is not the case due to the heat capacity of the unit itself, which means that it will not deliver the full heat flow in the first minutes after starting. However, we do not believe that this error significantly impacts the validity of the emulation.

The size of the tank is set to 750 liters, which is the size of the commercially available tank that the manufacturer suggests for a typical domestic installation. The heat demand $\dot{Q}_{\text {demand }}$
TABLE II

MICRO-CHP THERMAL PARAMETERS

\begin{tabular}{|l|c|}
\hline Storage tank capacity & $750 \mathrm{~L}$ \\
\hline Micro-CHP heat flow (when running) & $10.5 \mathrm{~kW}$ \\
\hline Heat demand (constant) & $5.25 \mathrm{~kW}$ \\
\hline Minimum heat storage temperature & $50^{\circ} \mathrm{C}$ \\
\hline Maximum heat storage temperature & $80^{\circ} \mathrm{C}$ \\
\hline
\end{tabular}

is assumed constant during the test, because the diurnal variation of outdoor temperature in winter is little, compared with the temperature difference between inside and outside. It is set to half the capacity of the micro-CHP unit, to emulate a winter day with an outdoor temperature around $0{ }^{\circ} \mathrm{C}$. In spring or autumn the variation is more significant and the demand generally lower. Table II summarizes the thermal parameters used for the micro-CHP test.

2) Control Action Decision: Based on the relative price, a decision must be made on what control actions to take, if any. The action can be to start, stop, or remain in the current state and if there were no constraints the decision could simply be to start when the relative price is above, e.g., 1 and stop if it drops below, e.g., 0 .

However, the generation is constrained by the possibility to dissipate the heat that is generated along with the electricity. The heat storage temperature must be kept between certain limits, to meet the heat demanded by the building and avoid boiling. Because this constraint affects the opportunity to react on future attractive prices, the storage temperature should generally be kept low when the price is low, to maximize the potential profit to make when the price increases and vice versa.

Finally, this particular micro-CHP unit has a minimum running time $t_{\text {on, min }}$ equal to $30 \mathrm{~min}$ for each start. To honor this, the controller will only stop the unit when it has been running for at least $30 \mathrm{~min}$, unless the maximum heat storage temperature is violated. Furthermore, it is only started when there is a certain margin to the maximum heat storage temperature.

The above considerations result in the decision diagram shown in Fig. 6. The parameter $k_{p}$ is the relative price at which the controller will fully charge the heat storage and is thus dimensionless. The value of $k_{p}$ affects the responsiveness to price changes and must be chosen carefully to optimize profit. It must be observed, that the value of the relative price time constant can affect the magnitude of the relative price (see Fig. 4), which effectively means that the time constant and $k_{p}$ must be tuned as a pair.

\section{Space Heating Thermostat}

The other design case is for the Devireg 550 thermostat by DEVI. It is used in connection with electric space heating systems and uses advanced filtering and adaptive control algorithms to compensate for disturbances and individual room characteristics. It contains an electromechanical relay that can switch the heater on or off and thereby shares the lacking possibility for continuous power adjustment with the micro-CHP unit.

The thermostat has an interface for a proprietary network type, which can be interfaced to the surroundings by means of 


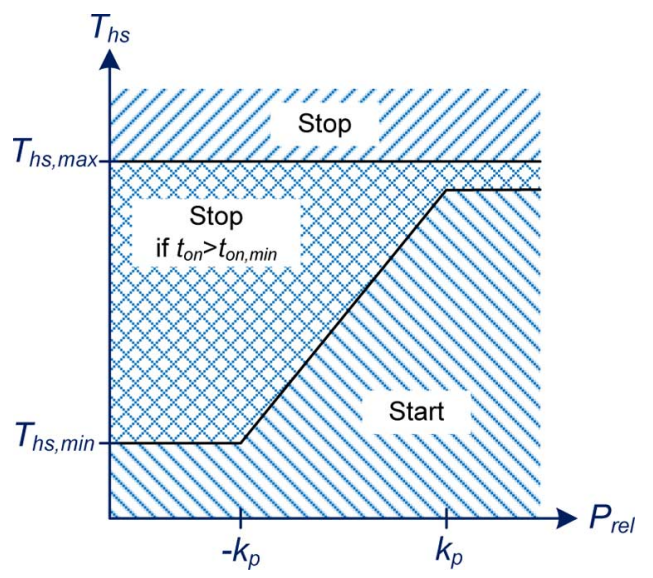

Fig. 6. Decision diagram for micro-CHP operation, depending on heat storage temperature, $T_{\mathrm{hs}}$ and relative price $P_{\text {rel }}$ and observing the operation time since last start, $t_{\text {on }}$, in relation to the minimum operation time per start, $t_{\text {on, min }}$. The parameter $k_{p}$ affects the responsiveness to price changes.

an RS232 adapter. This setup supports several thermostats on the same proprietary network, which can all be accessed using a single RS232 adapter. We use this interface to monitor and control the thermostat.

In the laboratory, we have used a model of a room that roughly corresponds to a $1: 10$ scale of all length measures. It consists of an expanded polystyrene box, inside which the thermostat is mounted, together with the heater, a fluorescent light bulb of 11 $\mathrm{W}$. To create a temperature difference to the surroundings, the box resides inside a large refrigerator during the tests. The heat capacity in the box is increased with by adding mass inside it.

This model is not a good representation of a real room, as the ratios between thermal masses and heat transfer rates are affected by scaling it down. For example, the thermal mass scales 1 to 1000 , whereas the heat conduction through the wall scales 1 to somewhere between 10 and 100 . This severely impacts the time constants involved in the heating process and consequently the dynamics of the price-responsive control, as will be shown in Section III.

1) Price-Responsive Control: Unlike the micro-CHP unit, the thermostat is not designed for direct external on-off control. The control parameter that is interfaced via the serial connection is the temperature setpoint, or rather an offset to the user's setpoint. This allows for an implementation close to the one in [2], except that we have chosen not to limit the offset, due to the use of the relative price that will tend to normalize towards zero offset, so extreme values will never persist. Consequently, the setpoint offset is set by

$$
T_{\text {offset }}=-k_{p} \cdot P_{\text {rel }}
$$

In this case $k_{p}$ is the temperature offset that will be set when the relative price is 1 and is thus expressed in degrees. This control method will not affect the average temperature, due to the definition of the relative price, but may cause temporary temperature deviations. This in turn may affect the comfort experienced by the end user, which means that the selection of $k_{p}$ in this case is not only a matter of finding an economical optimum, like for the micro-CHP unit, but rather a trade-off between comfort and economy.
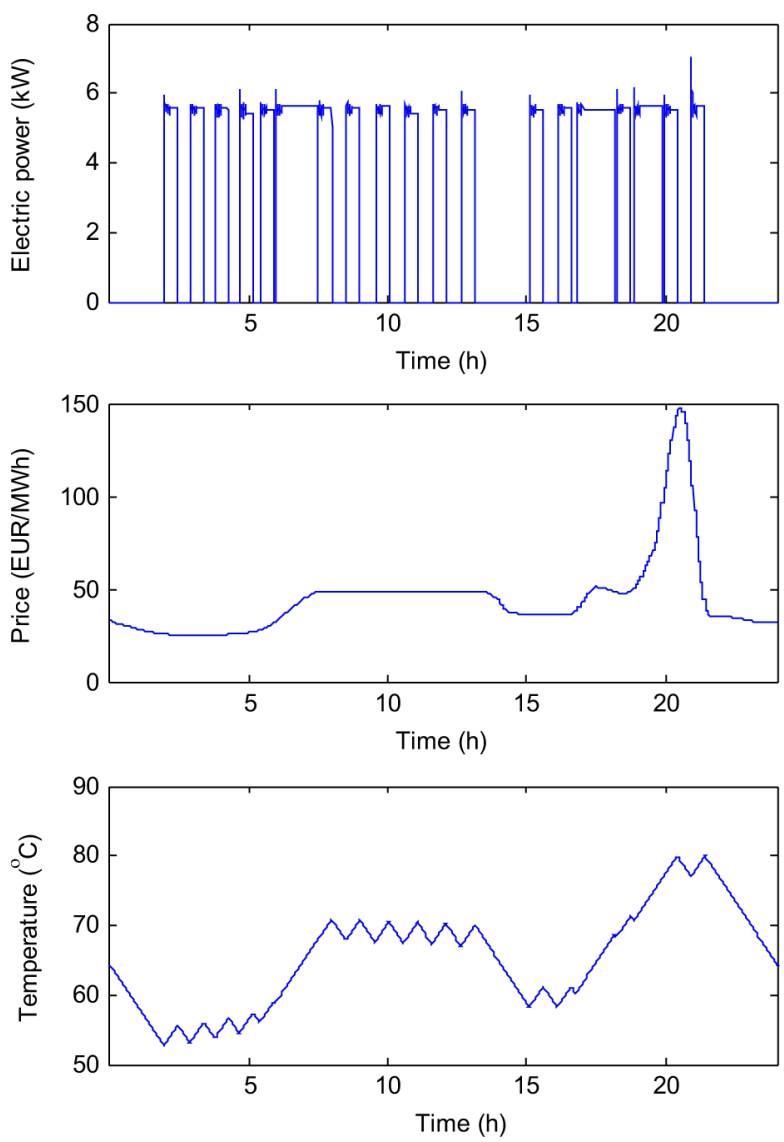

Fig. 7. Time series plots for micro-CHP operation. The temperature in the lower plot is the temperature of the emulated heat storage tank.

\section{RESULTS AND DiscuSSION}

We have conducted tests on both the micro-CHP unit and the space heating system and the results are summarized in Table III. Examples of observations are shown in Figs. 7 and 8.

With the used price series, the price-responsive controller reduces the electricity cost with $7.6 \%$ for the space heating setup and increases the income with $7.3 \%$ for the micro-CHP setup. For the latter, this happens without compromising the normal operation at all, whereas for the first, the consequence is a room temperature that varies several degrees over the course of a day. In numbers, the average is very close to the default operation with no price response, but the standard deviation of the temperature is $1.31^{\circ} \mathrm{C}$, compared with $0.65^{\circ} \mathrm{C}$ without price response.

This is mainly due to the fact that the laboratory model of the room is not representative, as described in Section II-D, which means that the temperature drops much faster than it would in a normal home. For this experiment a real home should be used, or a full-scale model in a climate chamber. However, the small model we have used still validates that the infrastructure and the price-responsive controller and interface to the thermostat, works altogether.

With regards to the micro-CHP operation, it is observed that when the price is ramping from about $6 \mathrm{~h}$ to $8 \mathrm{~h}$, the micro-CHP operates during the ramp, which means that the heat storage does not have much capacity left to let the micro-CHP operate during the long high-price period following the ramp. Apparently, this behavior is not optimal, but one must remember, that 

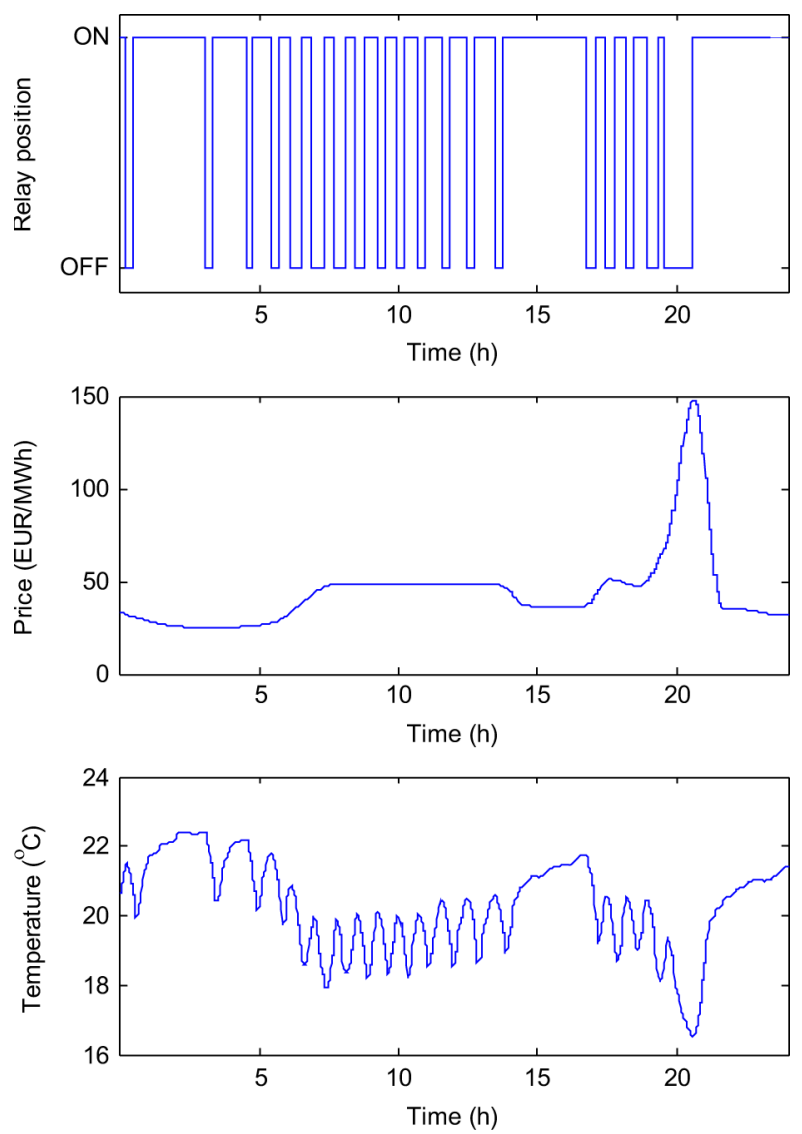

Fig. 8. Time series plots for space heating operation. The temperature in the lower plot is the temperature measured by the room thermostat of the model.

TABLE III

RESULTS SUMMARY

\begin{tabular}{|l|r|r|}
\hline & \multicolumn{1}{|c|}{ Micro-CHP } & Space heating \\
\hline$k_{p}$ & 1 & $1{ }^{\circ} \mathrm{C}$ \\
\hline Time constant $\tau$ & $12 \mathrm{~h}$ & $8 \mathrm{~h}$ \\
\hline $\begin{array}{l}\text { Electricity } \\
\text { generation/consumption }\end{array}$ & $64.5 \mathrm{kWh}$ & $191 \mathrm{Wh}$ \\
\hline $\begin{array}{l}\text { Average price earned/paid (Euro- } \\
\text { cent per kWh) }\end{array}$ & 4.78 & 4.11 \\
\hline Mean price (Euro-cent per kWh) & 4.45 & 4.45 \\
\hline Additional income/saving & $7.3 \%$ & $7.6 \%$ \\
\hline
\end{tabular}

the controller only knows the price 5 min ahead and has to optimize based on that. This is an example of how the day-ahead price schedule could have assisted in making a better decision, which is a topic we will address in future research.

To further investigate the performance of the controller with regards to optimizing the economics for the owner, one can compare with the best-case scenario: The controller knows the price for the full test period in advance and is not constrained by temperature limits. Then it is easy to calculate the theoretically best solution by operating the heater or the micro-CHP in the cheapest or most expensive periods respectively. The result is quite interesting - the electric heating would have saved $18 \%$ and the micro-CHP gained an additional $32 \%$ referenced to the base scenario with no price response at all. Comparing that with the obtained $7 \%$ in both cases with the relative price controller clearly illustrates that the algorithm could probably be improved. A more complex algorithm that considered the future forecasts and made an optimal schedule would most likely be more efficient; however, when considering these options, the lack of computing power in, e.g., a thermostat must be taken into account and the potential gain compared with the cost of implementing a more advanced thermostat.

Furthermore it is important to remember that knowing the future is utopia, as forecasts always have errors. In this case the large potential for additional earning by the micro-CHP is caused by a large peak that was not foreseen. It almost coincides with the day-ahead price peak, but that is very often not the case. In addition, not only the future price is uncertain in a real-life application. The heat demand is also not constant as in our tests and must be predicted to reach the optimal solution. And like with the price, the future will deviate from the predictions, so that the believed optimal scheduling turns out to be suboptimal. To fully uncover this problem and its solution, an excessive effort is needed to model the physics of the problem and simulate various algorithms under many different conditions in terms of prices, heat demand profiles and forecasts of both of them. We think that is an interesting topic for future research.

Another parameter that could possibly improve the control algorithm is the possibility to not let the units operate at full power. It may be more feasible to use a power setpoint rather than an energy (state-of-charge) setpoint. This would be possible if the machine could be throttled, or with applications that modulate the power with pulse width modulation. An example of this is the development in electric space heating systems, with heaters that operate in cycles as short as a few minutes, using pulsewidth modulation, or electric heat pumps with variable speed motor drives.

Looking from the system operator's point of view, this system provides more resources for system balancing that are comparable with the resources procured in the regulating power market, which in turn would reduce the cost for balancing. This cost is covered by those who cause imbalances, through the imbalance settlement. This is often intermittent generation, which with this system would have the imbalance penalties reduced. In the long run, this would make investment in renewable energy sources more attractive and we believe that the control-by-price concept is an important step towards the ambitious targets in that direction.

With this work, we have conceptually outlined a complete infrastructure that can realize the concept and in detail verified the operation of typical DER and flexible demand applications together with the price-responsive controller. The control principles that have been implemented and tested can easily be adapted to other applications.

\section{REFERENCES}

[1] F. C. Schweppe et al., "Homeostatic utility control," IEEE Trans. Power App. Syst., vol. PAS-99, pp. 1151-1163, May-Jun. 1980.

[2] D. Hammerstrom et al., Pacific Northwest GridWise Testbed Demonstration Projects, Part I. Olympic Peninsula Project National Technical Information Service, U.S. Dept. Commerce, 2007.

[3] F. L. Alvarado, "Is system control entirely by price feasible?," presented at the 2003 HICSS, Waikoloa, Hawaii.

[4] F. L. Alvarado, "Controlling power systems with price signals," Decision Support Syst., vol. 40, pp. 495-504, 2005.

[5] RFC-868, Time Protocol, Network Working Group, 1983. 
[6] Energy Management System Application Program Interface (EMS-API)-Part 301: Common Information Model (CIM) Base, IEC 61970-301, 2009,

[7] Energy Management System Application Program Interface (EMS-API)-Part 302: Common Information Model (CIM) Financial, Energy Scheduling and Reservations, IEC 61970-302, 2002, .

[8] Communication Networks and Systems for Power Utility Automation-Part 7-420: Basic Communication Structure-Distributed Energy Resources Logical Nodes, IEC 61850-7-420, 2009,

[9] A. B. Pedersen, E. B. Hauksson, P. B. Andersen, B. Poulsen, C. Træholt, and D. Gantenbein, "Facilitating a generic communication interface to distributed energy resources-Mapping IEC 61850 to RESTful services," in 1st IEEE Int. Conf. Smart Grid Commun. (SmartGridComm), 2010.

[10] Energinet.dk, download of market data [Online]. Available: http://www.energinet.dk/EN/El/The-wholesale-market/Download-of-market-data/Sider/default.aspx

Preben Nyeng (M'10) received the M.Sc. degree in industrial electrical engineering and the Ph.D. degree in power systems, from the Technical University of Denmark (DTU), Lyngby, in 2000 and 2011, respectively.

He was with Logos Design A/S from 2000 to 2006, developing embedded hardware and software systems and related database and communications systems. Since 2006 he has been with the Centre for Electric Technology at DTU, working in the field of smart grid, related information and communication technology and markets.

Dr. Nyeng is a member of CIGRE, the International Council on Large Electric Systems.
Jacob Østergaard (M'95-SM'09) received the M.Sc. degree in electrical engineering from the Technical University of Denmark (DTU), Lyngby, in 1995.

He was with Research Institute of Danish Electric Utilities for 10 years where he did research within power system transmission and distribution and was responsible for developing industrial-academic collaboration. Since 2005 he has been Professor and Head of Centre for Electric Technology, DTU. His research interests cover smart grids with focus on system integration of renewable energy and distributed energy resources, control architecture for future power system, and flexible demand.

Prof. Østergaard serves in several professional organizations, boards, and steering committees. He is head of the Danish experimental platform for electric power and energy, PowerLabDK, and he has been a member of the EU SmartGrids advisory council. In 2009 he received the IBM Faculty Award. 